\title{
AN ELECTROSTATIC PROBLEM IN BI-CYCLIDE COORDINATES*
}

\author{
BY K. AIKAWA (Yamanashi University, Kofu, Japan), \\ T. HISAMOTO (IBM, Japan, Fujisawa, Japan)
}

AND T. SUGANUMA (The Tohoku Communication Bureau, Sendai, Japan)

\begin{abstract}
This paper deals with an electrostatic problem for the field between two charged conductors $\pm u_{0}$ maintained at potential $\pm V$ in bi-cyclide coordinates $(u, v, \psi)$. In this coordinate system, Heine functions are used, of which something is known. Heine functions are the solutions of Heine differential equations. Though the problem is to be solved in the same manner as the problem in the case of bispherical coordinates, it has not been clarified because of the complexity of Heine functions. A Heine differential equation is solved to satisfy the boundary condition that the functions and their derivatives are bounded at the ends of interval, and eigenvalues and eigenfunctions are evaluated. A formula giving the capacity between two electrodes is presented and numerically calculated.
\end{abstract}

1. Introduction. This paper deals with an electrostatic problem for the field between two electrified conductors $\pm u_{0}$ maintained at potential $\pm V$, respectively, in bi-cyclide coordinates $(u, v, \psi)[1,2]$. Bi-cyclide coordinates are given when the two-dimensional plane in Fig. 1 is rotated about the $\zeta$-axis. We deal only with the axisymmetrical case. Though the problem is to be solved similarly to the problem in the case of bispherical coordinates [3], it has not been solved before because of the complexity of Heine functions, of which little is known. The eigenvalues and eigenfunctions for the Heine differential equation are obtained numerically in the same manner as in the solutions of SturmLiouville eigenvalue problem. A formula giving the capacity between two electrodes is presented and is numerically calculated.

2. Potential function. In the axisymmetric case, Laplace's equation is independent of the angle $\phi$, and is given as follows:

$$
\begin{aligned}
\nabla^{2} \phi=\frac{\Lambda^{3}}{a^{2} \Omega^{2} a n u^{2} d n u^{2} s n v c n v}\left\{s n v c n v \frac{\partial}{\partial u}\left[\frac{c n u d n u}{\Lambda} \frac{\partial \phi}{\partial u}\right]\right. \\
+c n u d n u \frac{\partial}{\partial v}\left[\frac{s n v c n v}{\Lambda} \frac{\partial \phi}{\partial v}\right]=0, \quad-K \leq u \leq K, \quad 0 \leq v \leq K^{\prime},
\end{aligned}
$$

where

$$
\begin{aligned}
\Lambda & =1-d n^{2} u s n^{2} v, \\
\Omega^{2} & =\left(1-s n^{2} u d n^{2} v\right)\left(d n^{2} v-k^{2} s n^{2} u\right),
\end{aligned}
$$

* Received February 25, 1976. The authors wish to express their gratitude to Profs. M. Kurihara and C. Ito and to M. Takahara and H. Naruge for their help. 


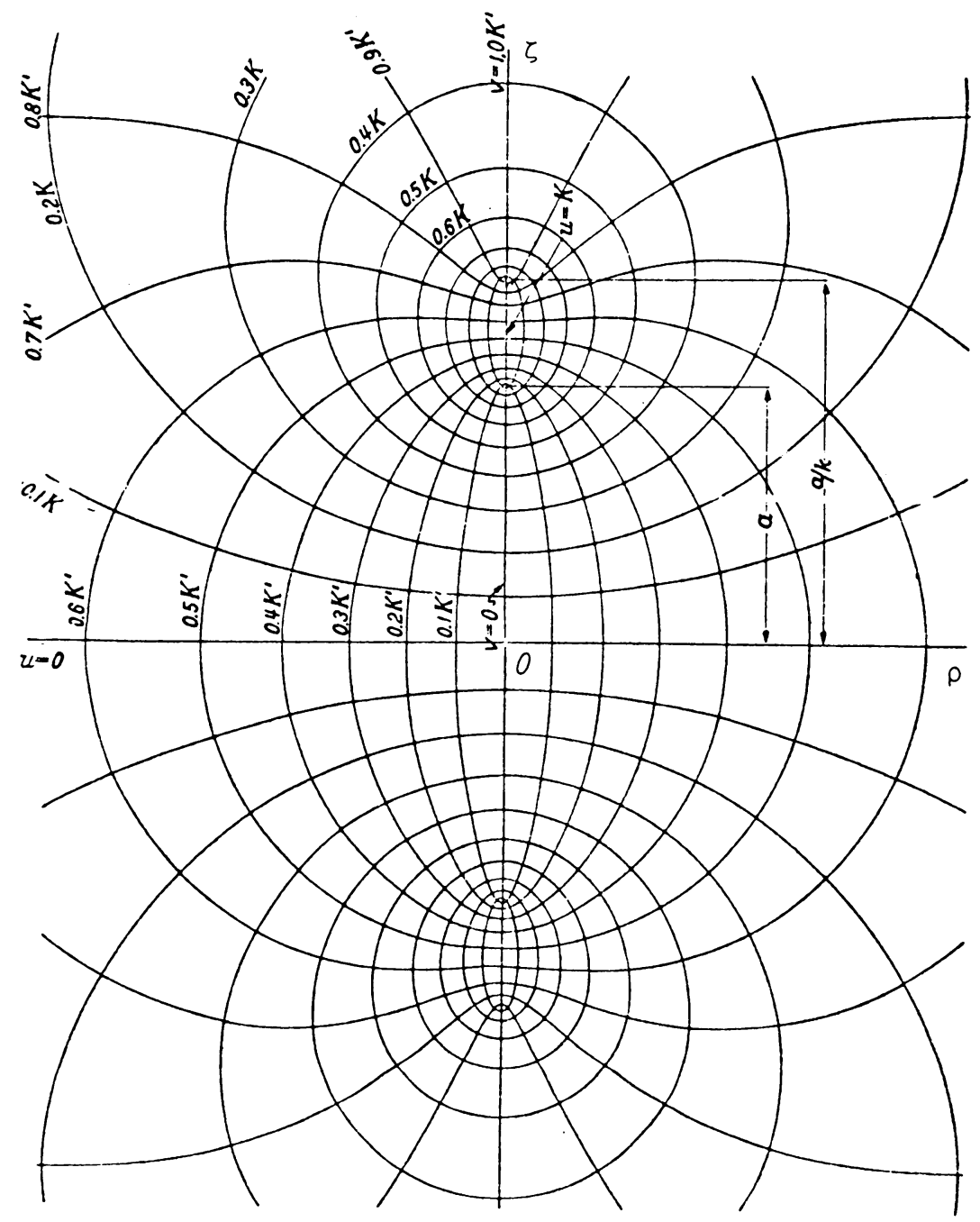

FIG. 1. Two-dimensional plane of bi-cyclide coordinates.

a is the distance from origin to focus, $k$ is the modulus of the Jacobian elliptic functions $\operatorname{sn}(u, k)$ and $\operatorname{sn}\left(v, k^{\prime}\right)\left[k^{\prime 2}=1-k^{2}\right]$, for which $s n u$ and $s n v$ are the abbreviations respectively, and $c n u, c n v, \ldots$ are similar abbreviations.

Suppose that Eq. (1) has a solution of the form

$$
\phi=\Lambda^{1 / 2} M(u) N(v)
$$

and let $\alpha$ be a separation constant; then the Laplace equation (1) is separated into

$$
\begin{gathered}
\frac{d^{2} M}{d u^{2}}-\frac{s n u\left(d u^{2} u+k^{2} c n^{2} u\right)}{c n u d n u} \frac{d M}{d u}+\left(2 k^{2} s n^{2} u-\alpha\right)=0, \\
\frac{d^{2} N}{d v^{2}}+\frac{s n u\left(c n^{2} v s n^{2} v\right)}{s n v c n v} \frac{d N}{d v}+\left(-2 d n^{2} v+\alpha\right)=0
\end{gathered}
$$


Substitution of $s n^{2} u=z$ into (3) and $d n^{2} v=z$ into (4) reduces both to the canonical form

$$
\frac{d^{2} Z}{d z^{2}}+\frac{1}{2}\left(\frac{1}{z}+\frac{2}{z-1}+\frac{2}{z-c}\right) \frac{d Z}{d z}+\frac{2 z-\beta c}{4 z(z-1)(z-c)} Z=0
$$

where $1 / k^{2}=c, \beta=\alpha$ for (3) and $k^{2}=c, \beta=\alpha / k^{2}$ for (4). Eqs. (3) and (4) are the Jacobian form and (5) is the algebraic form of the Heine equation, whose solutions are Heine functions. Eq. (5) has four singular regular points $0,1, c$, and $\infty$ and has series solutions expanded about these singular points.

Linearly independent particular solutions of (3) expanded about $z=s n^{2} u=0$ are as follows:

$$
\begin{aligned}
& U_{p}\left(k, s n^{2} u\right)=s n u \sum_{j=0}^{\infty} c_{j} s n^{2 j} u, \\
& V_{p}\left(k, s n^{2} u\right)=\sum_{j=0}^{\infty} c_{j} s n^{2 j} u, \quad j=0,1,2, \cdots
\end{aligned}
$$

where $p^{2}=\alpha$. Particular solutions of (4) expanded about $z=d n^{2} v=1$ are as follows:

$$
\begin{aligned}
& U_{p^{\prime}}\left(1 / k, d n^{2} v\right)=\sum_{j=0}^{\infty} c_{j}\left(d n^{2} v-1\right)^{j} \\
& V_{p^{\prime}}\left(1 / k, d n^{2} v\right)=U_{p^{\prime}}\left(1 / k, d n^{2} v\right) \ln \left(1-d n^{2} v\right)+\sum_{j=0}^{\infty} d_{j}\left(1-d n^{2} v\right)^{j}
\end{aligned}
$$

where $p^{\prime}=p / k$. The coefficients $c_{j}$ and $d_{j}$ are determined uniquely in terms of $r$ (the roots of the indicial equation), $k(0<k<1)$, and $\alpha$ for the expansion points and they are evaluated by computer. For simplicity, (6), (7), (8) and (9) are abbreviated to $U_{p}(u), V_{p}(u)$, $U_{p}^{\prime}(v)$ and $V_{p}^{\prime}(v)$, respectively.

The general solution of $(3)$ is the linear combination of the two particular solutions $U_{p}(u)$ and $V_{p}(u)$ :

$$
M(u)=A U_{p}(u)+B V_{p}(u)
$$

and similarly for (4):

$$
N(v)=C U_{p}^{\prime}(v)+D V_{p}^{\prime}(v),
$$

where $A, B, C$ and $D$ are arbitrary constants.

When the potential is maintained as

$$
\begin{gathered}
\phi= \pm V \text { at } u= \pm u_{0}, \\
\phi=0 \text { at } u=0,
\end{gathered}
$$

$\phi$ must be an odd function of $u$. In order to exclude $U_{p}(u)$ which is an even function of $u, B$ must be zero. The potential must be bounded. Since $V_{p^{\prime}}(v)$ includes $\ln (0), D$ must be zero in order to exclude $V_{p^{\prime}}(v)$. Hence, we assume the solution to be as follows:

$$
\phi=\Lambda^{1 / 2} \sum_{j=0}^{\infty} A_{n} \frac{U_{p_{n}}(u)}{U_{p_{n}}\left(u_{0}\right)} U_{p_{n}{ }^{\prime}}(v), \quad n=0,1,2, \cdots,
$$

where $A_{n}$ should be determined so to satisfy necessary boundary conditions. To obtain $A_{n}$, we utilize the orthogonality of the function $U_{p_{n}{ }^{\prime}}(v)$. 
Considering a set of $U_{p_{n^{\prime}}}(v)$ which is orthogonal with respect to weighting function $r(v)$ on the interval $\left[0, K^{\prime}\right]$, we have

$$
A_{n}=\frac{V \int_{0}^{K^{\prime}} \frac{U_{p_{n^{\prime}}}(v) r(v)}{\left(1-d n^{2} u_{0} s n^{2} v\right)^{1 / 2}} d v}{\int_{0}^{K^{\prime}}\left[U_{p_{n^{\prime}}}(v)\right]^{2} r(v) d v}=\frac{V \int_{0}^{K^{\prime}} \frac{U_{p_{n^{\prime}}}(v) r(v)}{\left(1-d n^{2} u_{0} s n^{2} v\right)^{1 / 2}} d v}{\left\|U_{p_{n^{\prime}}}(v)\right\|^{1 / 2}},
$$

where $\left\|U_{p_{n^{\prime}}}(v)\right\|^{2}$ is the weighted norm of the form

$$
\left\|U_{p_{n^{\prime}}}(v)\right\|^{2}=\int_{0}^{K^{\prime}}\left[U_{p_{n^{\prime}}}(v)\right]^{2} r(u) d v .
$$

The weighting function $r(u)$ will be given in Sec. 3 .

3. Determination of eigenvalues. It is necessary to determine the eigenvalues and eigenfunctions for (3) or (4). An advanced problem for the determination of eigenvalues appears in the case of bispherical coordinates. In this problem, eigenvalues are determined under the boundary conditions that the solutions should be bounded at the ends of the interval $x[-1,1]$ or $\theta[\pi, 0]$ which are the regular singular points; they are given by $\lambda_{n}=n(n$ $+1), n=0,1,2, \cdots$ (integers) [3]. The corresponding eigenfunctions are Legendre polynomials $P_{n}(x)=P_{n}(\cos \theta)$. If $n$ is not an integer, the solutions are not eigenfunctions and diverge at $x=-1$.

We proceed as follows. The Legendre equation is replaced by the Heine equation (4).

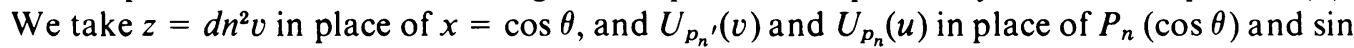
$h\left[\left(n+\frac{1}{2}\right) \eta\right]$, respectively. We must determine the eigenvalues in the space between the two conductors in bi-cyclide coordinates. They are obtained in the same manner as in the solution of the Sturm-Liouville eigenvalue problem.

The general form of the second-order Sturm-Liouville problem consists of a differential equation

$$
\frac{d}{d v}\left[p(v) \frac{d y}{d v}\right]+[-q(v)+\lambda r(v)] y=0,
$$

on the interval $a \leq u \leq b$, together with the boundary conditions

$$
\begin{aligned}
\alpha_{1} y(a)+\alpha_{2} y^{\prime}(a) & =0, \\
\beta_{1} y(b)+\beta_{2} y^{\prime}(b) & =0
\end{aligned}
$$

at the endpoints.

Let $\lambda_{n}(n=0,1,2, \ldots)$ be the eigenvalues and $y_{n}$ the eigenfunctions which build up orthogonal series on the interval $[a, b]$ with respect to the weighting function $r(u)$. If

$$
\left\|p(v) y y^{\prime}\right\|_{a}^{b}=0
$$

holds, then the eigenvalues and the eigenfunctions are given by

$$
\lambda_{n}=\frac{\int_{a}^{b}\left[p(v) y_{n}{ }^{2}+q(v) y_{n}^{2}\right] d v}{\int_{a}^{b} r(u) y_{n}^{2} d v} .
$$


The values of $\lambda_{n}$ and $y_{n}$ that satisfy (20) are calculated by an iterative process, though this is tedious.

Eq. (4) can be expressed in Sturm-Liouville form as

$$
\frac{d}{d v}\left(\operatorname{snv} c n v \frac{d N}{d v}\right)-\left(2 d n^{2} v+\alpha\right) \operatorname{snv} c n v N=0 .
$$

Comparison of (16) and (21) gives the following relation:

$$
y=N(v), \quad p(v)=\operatorname{snv} c n v, \quad q(v)=2 \operatorname{snv} c n v d n^{2} v, \quad r(v)=\operatorname{snv} c n v, \quad \lambda=-\alpha .
$$

Let the boundary conditions be

$$
N(v)=1, \quad N^{\prime}(v)=0 \quad \text { at } \quad v=0, \quad N(v) \text { bounded, } \quad N^{\prime}(v)=0 \text { at } \quad v=K^{\prime} ;
$$

then (19) holds and (20) becomes

$$
\lambda_{n}=\frac{\int_{0}^{K^{\prime}} \operatorname{snv} c n v\left(N_{n}{ }^{2}-2 d n^{2} v N_{n}^{2}\right) d v}{\int_{0}^{K^{\prime}} \operatorname{snv} c n v N_{n}^{2} d v} .
$$

The eigenvalues and eigenfunctions which satisfy (24) are computed. Examples of them are shown in Fig. 2. Another method is to calculate the eigenvalues by the Rayleigh-Ritz variational method and then obtain $U_{p_{n}}(v)$ from $(8)$. The eigenvalues calculated by the Rayleigh-Ritz method agree closely with those obtained by the iterative method.

4. Expression for capacity. Let $\epsilon$ be the dielectric constant of the medium, $E$ be the electric field intensity over the surface of the electrode and $d \mathrm{~A}$ be an element of area. Then the total charge $Q$ over the electrode is given by

$$
Q=\int_{S} \epsilon \mathrm{E} d \mathrm{~A}
$$
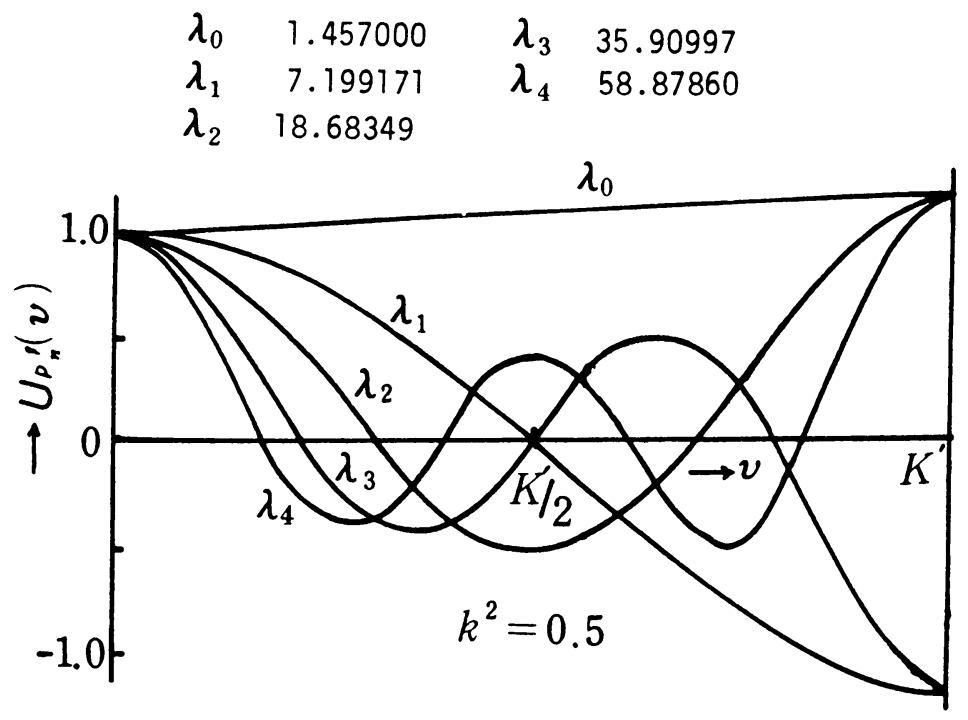

FIG. 2. Heine eigenfunctions $U_{p_{n}{ }^{\prime}}(\nu)$. 
Division of (25) by $2 \mathrm{~V}$ gives the capacity $C$ and division of the capacity by $a$ gives the normalized capacity

$$
\frac{C}{a}=\frac{Q}{2 a V}=\frac{\int_{S} \epsilon \mathrm{E} d \mathrm{~A}}{2 a V}
$$

In the axisymmetric case

$$
\mathrm{E}=-\operatorname{grad} \phi=-\frac{\Lambda}{a \Omega}\left(\mathrm{a}_{u} \frac{\partial \phi}{\partial u}+\mathrm{a}_{v} \frac{\partial \phi}{\partial v}\right),
$$

where $\mathrm{a}_{u}$ and $\mathrm{a}_{v}$ are unit vectors, and

$$
d \mathrm{~A}=\mathrm{a}_{u}\left(g_{22} g_{33} d v d \psi\right)^{1 / 2},
$$

where $g_{22}, g_{33}$ are metric coefficients given by [1]:

$$
g_{22}=\frac{a^{2} \Omega^{2}}{\Lambda^{2}}, \quad g_{33}=\frac{a^{2}}{\Lambda^{2}} c n^{2} u d n^{2} u s n^{2} v c n^{2} v .
$$

Substitution of (27), (28) and (29) into (26) gives

$$
\frac{C}{a}=\frac{\pi \epsilon}{V} \int_{0}^{K^{\prime}}\left(\frac{1}{\Lambda} \frac{\partial \phi}{\partial u} c n u d n u \text { vnc cnv }\right)_{u=u_{0}} d v .
$$

From (13), we have

$$
\begin{aligned}
\left.\frac{\partial \phi}{\partial u}\right|_{u=u_{0}}= & k^{2} s n u^{2} c n u^{2} d n u^{2} s n^{2} v\left(1-d n^{2} u_{0} s n^{2} v\right)^{-1 / 2} \sum_{n=0}^{\infty} A_{n} U_{p_{n^{\prime}}}(v) \\
& +\left(1-d n^{2} u_{0} s n^{2} v\right)^{1 / 2} \sum_{n=0}^{\infty} A_{n} \frac{U_{p_{n^{\prime}}}\left(u_{0}\right)}{U_{p_{n}}\left(u_{0}\right)} U_{p_{n^{\prime}}}(v),
\end{aligned}
$$

where

$$
U_{p_{n^{\prime}}}\left(u_{0}\right)=\left.\frac{\partial}{\partial u} U_{p_{n}}(u)\right|_{u=u_{0}} .
$$

Substitution of (31) into (30) gives

$$
\begin{aligned}
\frac{C}{a}= & \frac{\pi \epsilon c n u_{0} d n u_{0}}{V} \sum_{n=0}^{\infty} A_{n} \int_{0}^{K^{\prime}} \frac{s n v c n v}{\left(1-d n^{2} u_{0} s n^{2} v\right)^{1 / 2}} U_{p_{n^{\prime}}}(v) \\
& \cdot\left[\frac{k^{2} s n u_{0} c n u_{0} d n u_{0} s n^{2} v}{1-d n^{2} u_{0} s n^{2} v}+\frac{U_{p_{n^{\prime}}}\left(u_{0}\right)}{U_{p_{n}}\left(u_{0}\right)}\right] d v,
\end{aligned}
$$

where $A_{n}$ is given by (14).

The approximate values of $C / a$ calculated by (32) are listed in Table 1 , where $u_{0} / K$ relates the shape of the electrodes and $C_{n} / a$ denotes a partially normalized capacity corresponding to $n$. The contribution of $C_{0} / a$ to $C / a$ is dominantly large and the contributions of $C_{1} / a, C_{2} / a, \cdots$ are very small. This property is more remarkable when the values of $u_{0} / K$ and $k$ are larger. The approximate values previously obtained [4] agree with the values evaluated by this exact method (within reasonable error). The relation of $C / a$ to $u_{0} / K$ is shown for the parameters $k^{2}=0.1,0.3, \ldots 0.9$ in Fig. 3. 
TABLE 1. Normalized capacity, $k^{2}=0.1, \epsilon=8.8552 \times 10^{-12}[\mathrm{~F} / \mathrm{m}]$.

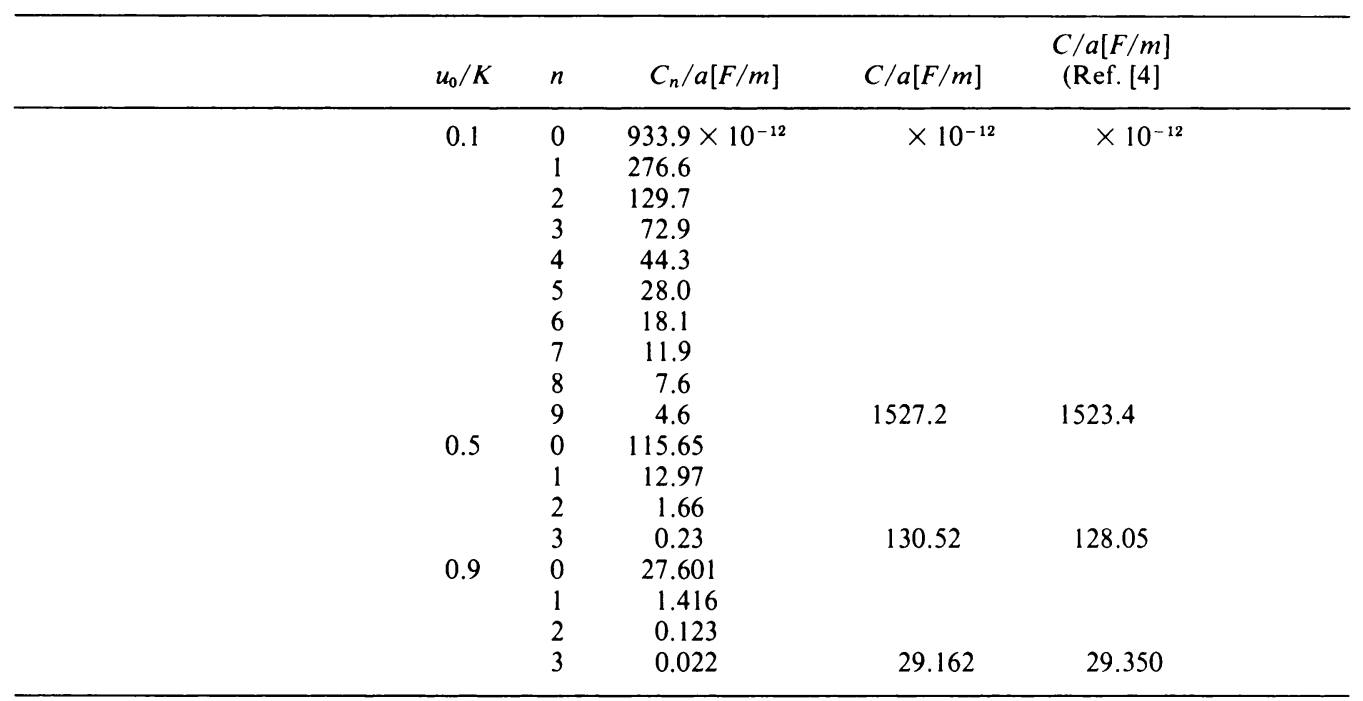

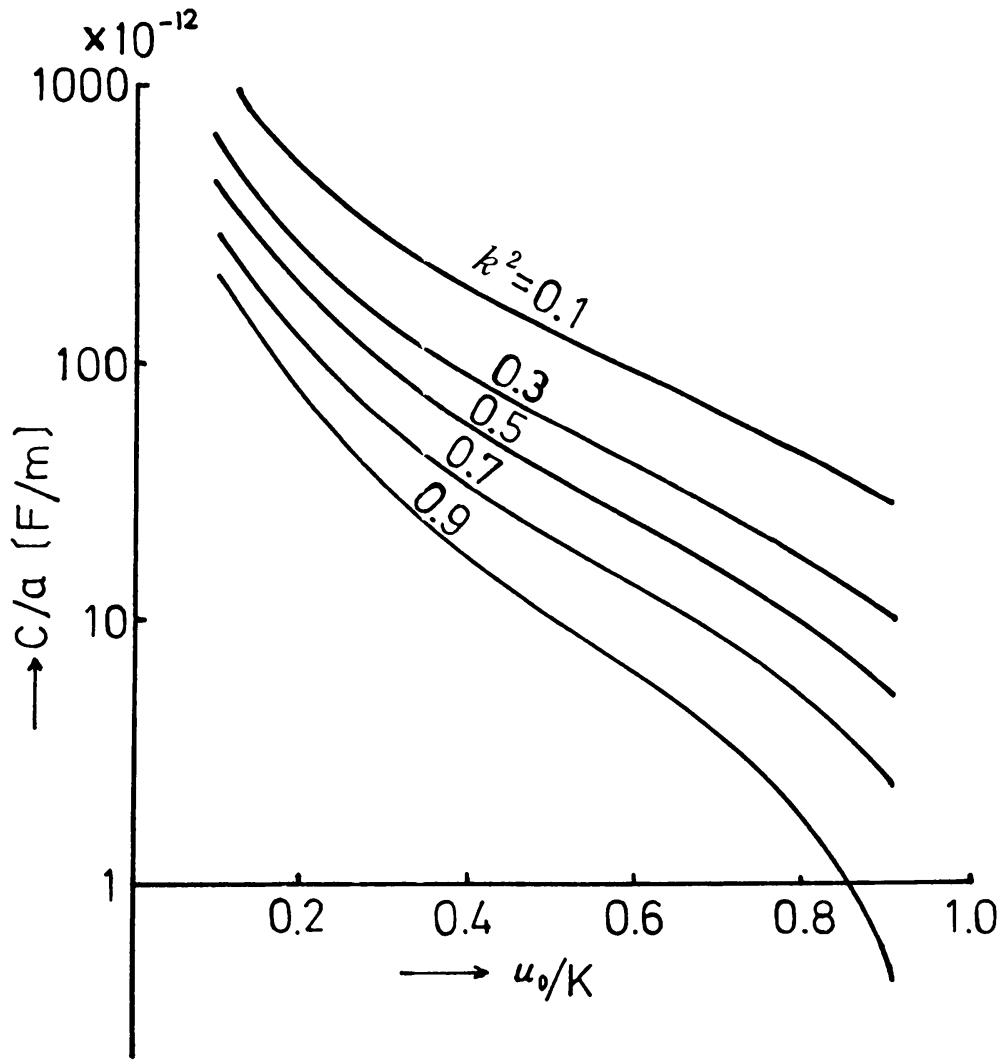

FIG. 3. Normalized capacity. 
5. Concluding remarks. The main theme of this paper has been the numerical calculation of Heine functions and the capacity between two electrodes $\pm u_{0}$ in bi-cyclide coordinates. The eigenvalues and eigenfunctions are not represented in simple functional relation as for Legendre polynomials, and their evaluation is possible with a huge amount of computation. While electrostatic problems in the case of bispherical coordinates are solved, problems in bi-cyclide coordinates have not been clarified because of the complexity of Heine functions. In solving the boundary-value problem for Heine differential equations, an electrostatic problem has been solved and the capacity is evaluated. This paper presents a new contribution to the hitherto unknown field of special functions, eigenvalue problems and engineering applications, though the problem belongs to classic mathematics.

The computation was carried out by the double precision method, restricting the maximum numbers of terms to 200 by use of Facom 270-30 computer.

\section{REFERENCES}

[1] P. Moon and D. E. Spencer, Field theory handbook, Springer, Berlin, 1961

[2] C. Ito and K. Aikawa, Heine oyobi Wangerin no kansū no sūchi keisan (Numerical calculations of Heine and Wangerin functions), Report of Faculty of Engineering, Yamanashi University, No. 17, 91-98 (1963)

[3] P. Moon amd D. E. Spencer, Field theory for engineers, D. V an Nostrand Co. Inc., Princeton, 1961

[4] K. Aikawa and Y. Ohki, An approximate formula giving the capacity between two spindle-shaped electrodes placed in rotational symmetry on a straight line, Report of Faculty of Engineering, Yamanashi University, No. 14, 93-100 (1963) 